\title{
Isolation of Human Transcripts Expressed in Hamster Cells from YACs by cDNA Representational Difference Analysis
}

\author{
Jessie Gu, ${ }^{1,3}$ Xin-Yuan Guan, ${ }^{2}$ and Melissa A. Ashlock ${ }^{1,4}$ \\ ${ }^{1}$ Genetics and Molecular Biology Branch and ${ }^{2}$ Cancer Genetics Branch, National Human Genome Research Institute, \\ National Institutes of Health, Bethesda, Maryland 20892-4442 USA
}

\begin{abstract}
Gene isolation methods used during positional cloning rely on physical contigs consisting of bacterial artificial chromosomes, $\mathrm{Pl}$, or cosmid clones. However, in most instances, the initial framework for physical mapping consists of contigs of yeast artificial chromosome (YACs), large vectors that are suboptimal substrates for gene isolation. Here we report a strategy to identify gene sequences contained within a YAC by using cDNA representational difference analysis (RDA) to directly isolate transcripts expressed from the YAC in mammalian cells. The RDA tester cDNAs were generated from a previously reported hamster cell line derived by stable transfer of a 590-kb YAC (911D5) that expressed NPCl, the human gene responsible for Niemann-Pick type C (NP-C). The driver cDNAs were generated from a control hamster cell line that did not contain the YAC that expressed NPCl. Among the gene fragments obtained by RDA, NPC1 was the most abundant product. In addition, two non-NPC1 fragments were isolated that were mapped to and expressed from 911D5. One of these RDA gene fragments (7-R) spans more than one exon and has $98 \%$ sequence identity with a human cDNA clone reported previously as an expressed sequence tag (EST), but not mapped to a chromosomal region. The other fragment (2-R) that had no significant sequence similarities with known mammalian genes or ESTs, was further localized to the region of overlap between YACs 911D5 and 844E3. The latter YAC is part of a contig across the NP-C candidate region, but does not contain NPCl. This two-part approach in which stable YAC transfer is followed by cDNA RDA should be a useful adjunct strategy to expedite the cloning of human genes when a YAC contig is available across a candidate interval.
\end{abstract}

[The sequence data described in this paper have been submitted to GenBank under accession nos. AF117641 and AF117642.]

The identification of genes responsible for inherited human disorders is an integral part of the Human Genome Project. There are several widely applied methods for gene isolation associated with conventional positional cloning, including a combination of sequencing, database searching and candidate gene analysis, exon trapping (Duyk et al. 1990; Buckler et al. 1991; Krizman and Berget 1993), and direct selection (Lovett 1994; Osborne-Lawrence et al. 1995; Simmons et al. 1995; Del Mastro and Lovett 1997). The effective use of most of these methods relies on physical contigs constructed from genomic clones contained within vectors of manageable sizes, such as bacterial artificial chromosomes (BACs), P1 clones, or cosmids. However, in most positional cloning efforts, the initial framework for physical mapping consists of contigs of yeast artificial chromosomes (YACs). Unfortunately, these large vectors are suboptimal substrates for most of the commonly used gene isolation techniques listed above.

Here we report a novel strategy designed to accel-

\footnotetext{
${ }^{3}$ Present address: Genome Therapeutics Corporation, Department of $\mathrm{Hu}$ man Genetics, Waltham, Massachusetts 02154 USA. ${ }^{4}$ Corresponding author.

E-MAIL melis@nhgri.nih.gov; FAX (301) 492-4929.
}

erate gene identification, which was developed with resources from the positional cloning of NPC1, the gene responsible for Niemann Pick Type C [(NP-C), Carstea et al. 1997]. In the process of identifying NPC1, the candidate interval was substantially narrowed by complementation of the NP-C phenotype by stable integration of a 590-kb YAC (911D5) into the genome of CT60, an NP-C cell line (Gu et al. 1997) derived by chemical mutagenesis of the Chinese hamster ovary (CHO) cell line, 25-RA, which has a normal phenotype with respect to NP-C (Cadigan et al. 1990). One advantage of this successful YAC complementation cloning strategy was that the search for candidate genes was quickly focused on only a few hundred kilobase of DNA. However, it was still necessary to generate smaller insert contigs across the narrowed interval to identify NPC1 (Carstea et al. 1997). To preclude this time-consuming step in future studies, we sought to demonstrate that YACs such as 911D5 could be used directly for the isolation of human transcripts. The current study combines YAC complementation cloning with cDNA representational difference analysis (RDA), a technique that relies on the generation of tester and driver representations from cDNAs generated from two types of cell populations expected to differentially ex- 
press specific transcripts (Lisitsyn et al. 1993; Hubank and Schatz 1994; Braun et al. 1995; Chu and Paul 1997; Gress et al. 1997). RNA from the NPC1 complemented cell lines in which YAC 911D5 was stably integrated (911D5A1 and 911D5A13) was used to generate the tester representations, and a noncomplemented hamster cell line (CFTRA1) was used to generate the driver. Because RDA eliminates fragments present at similar concentrations in both populations, leaving only the differences, NPC1 and other genes expressed from 911D5 should be identified among the endogenous hamster transcripts in the complemented cell lines.

\section{RESULTS}

\section{Generation of First and Second Difference Products}

The cDNA RDA procedure used in the present study was based closely on the protocol described by Hubank and Schatz (1994). Our first goal was to use RDA to identify NPC1, known to be on YAC 911D5. Tester cDNAs were synthesized separately from two clonal cell lines derived by spheroplast fusion of 911D5 with CT60 cells. Both of the clonal cell lines, 911D5A1 and 911D5A13, express NPC1 at a level detectable by Northern blot hybridization ( $\mathrm{Gu}$ et al. 1997). The driver cDNA was synthesized from a control clonal cell line (CFTRA1) that does not express NPC1, derived by spheroplast fusion of CT60 with a 325-kb YAC that contains the cystic fibrosis transmembrane conductance regulator gene [(CFTR), Mogayzel et al. 1996; Gu et al. 1997]. Each of the cell lines expresses a gene $\left(\right.$ neo $\left.^{r}\right)$ that confers resistance to the neomycin analog, G-418. Tester and driver cDNAs were digested with the restriction enzyme DpnII to generate small fragments (mean length $256 \mathrm{bp}$ ) that should be easily amplified by PCR. Because there are 10 DpnII sites in the full-length NPC1 cDNA generating fragments ranging from 37-1200 bp, NPC1 should be readily amplifiable by the PCR conditions used. Tester and driver cDNA representations were generated by PCR and then denatured and reassociated to generate the first difference products (DP I). The procedure was repeated to generate the second difference products (DP II).

The difference products (I and II) were initially evaluated by agarose gel electrophoresis (Fig. 1A). The complexity of DP I was reduced compared with the amplicons from tester cDNAs from 911D5A1 or 911D5A13. The complexity was further reduced in DP II as indicated by the distinct bands that appeared on the ethidium bromide-stained gel. The staining pattern of the difference products obtained by use of either 911D5A1 or $911 D 5 \mathrm{~A} 13$ as the tester cDNAs looked similar, consistent with the likelihood that these two clonal cell lines are sibling clones (Gu et al. 1997).

A probe derived from the full-length NPC1 cDNA (Carstea et al. 1997) hybridized to the original DpnII
A

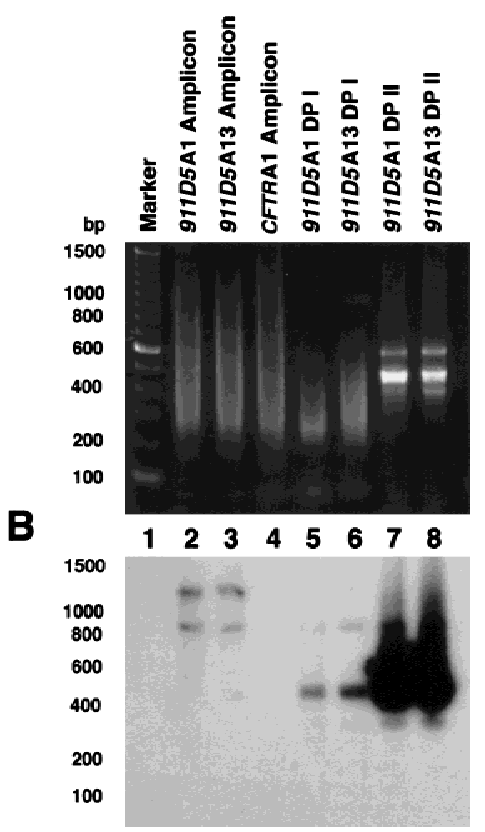

Figure 1 Analysis of difference products obtained by RDA. RDA was carried out with cDNA from the complemented cell lines 911D5A1 or 911D5A13 as testers and CDNA from the control cell line CFTRA1 as the driver. (A) Difference products were run on a $1.5 \%$ agarose gel that was stained with ethidium bromide. Representation amplicons and first and second difference products (DP I and DP II, respectively) are shown. (B) Southern blot of the gel shown in $A$, hybridized with a ${ }^{32} \mathrm{P}$-labeled probe generated by random priming of the full-length NPC1 CDNA.

digested amplicons from both tester cDNAs, but not to the driver cDNA (Fig. 1B, cf. lanes 2 and 3 with lane 4). The same probe hybridized with progressively stronger intensity to the DP I and DP II, respectively, derived from the cDNA from both testers (Fig. 1B, lanes 5-8), indicating that NPC1 is enriched in the difference products. The smaller fragments appear to be preferentially enriched after each round of subtraction and amplification. This result is consistent with the use of PCR conditions that are likely to favor smaller fragments during the generation of the difference products. These data demonstrate that RDA can be used to detect a gene expressed as a consequence of integrated heterologous DNA. We attempted to distinguish the specific bands predicted for DpnII digestion of the NPC1 cDNA by altering gel running conditions and/or film exposure time, but were unsuccessful.

\section{Cloning the Second Difference Products}

The second goal of the study was to isolate transcripts expressed from genes other than NPC1 that might be contained on YAC $911 D 5$ (summarized in Fig. 2). Because NPC1 spans $<100 \mathrm{~kb}$ of this $590-\mathrm{kb}$ YAC (J.A. Morris and E.D. Carstea, unpubl.), we expected that the YAC could contain up to 10-20 additional genes 
Gu et al.

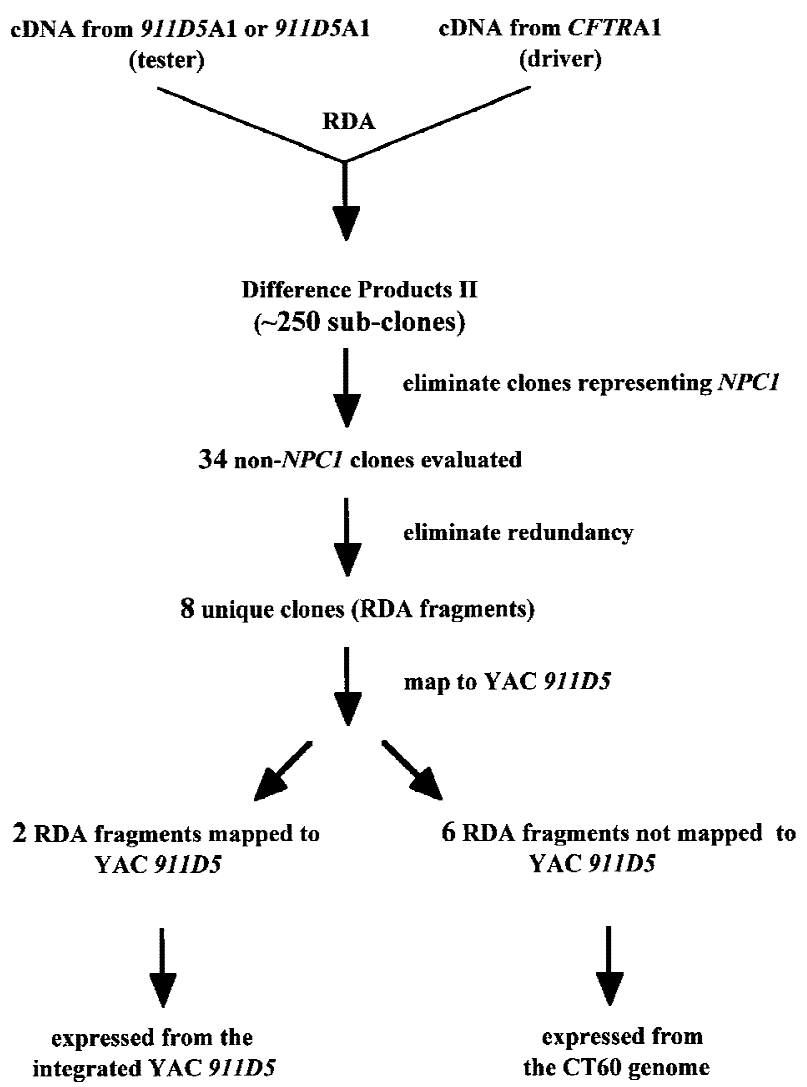

Figure 2 Summary of the isolation and analysis of unique nonNPC1 gene fragments identified by RDA.

(assuming an average gene density of one gene per 30 $\mathrm{kb})$. First, the gene fragments represented in DP II were subcloned and colony lifts were prepared and screened with the full-length NPC1 cDNA probe. The majority (>75\%) of the RDA gene fragments represented by the recombinant colonies hybridized to the NPC1 probe. Then, the RDA gene fragments isolated from 911D5A1 and 911D5A13 (12 and 22 colonies, respectively) that did not hybridize to NPC1 were further evaluated (Fig. 2). To eliminate redundancy among the non-NPC1 RDA gene fragments, two fragments were randomly chosen (one each from the DP II from 911D5A1 and 911D5A13) as probes to hybridize to an arrayed panel of the 34 non-NPC1 colonies. This procedure reduced the number of nonredundant RDA gene fragments to 11. Sequence analysis revealed that 7 of these 11 fragments were unique, 3 others were redundant with each other, and 1 fragment yielded poor quality sequence data. On the basis of this data, PCR primers were synthesized for the 8 unique gene fragments.

\section{Gene Fragment Assessment: Localization on YAC 911D5, Expression and Sequence Analysis}

On the basis of the premise of the cDNA RDA procedure, the eight fragments were obtained because they were present to a greater extent in the cDNA of the tester compared with that of the driver. The next step was to determine if the differential expression of these eight fragments was a direct consequence of expression of a gene contained within YAC 911D5. First, the primer pairs designed for each of the eight unique gene fragments were used in PCR assays to determine whether the fragments mapped back to the YAC. Two of the eight RDA gene fragments (2-R and 7-R; GenBank accession nos. AF117641 and AF117642, respectively) were found to map back to YAC 911D5 (Figs. $2-4)$. For 2-R, a PCR product of the expected size (261 bp) was amplified from genomic DNA from YAC 911D5 and the cell lines in which 911D5 was stably integrated [the testers 911D5A1 and 911D5A13 and another NPC1 complemented cell line, 911D5B5 (Gu et al. 1997)]. No product was amplified from genomic DNA from the parental cell lines CT60 and 25-RA. In-

\section{A}

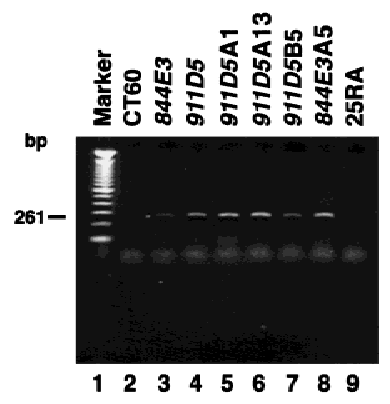

B

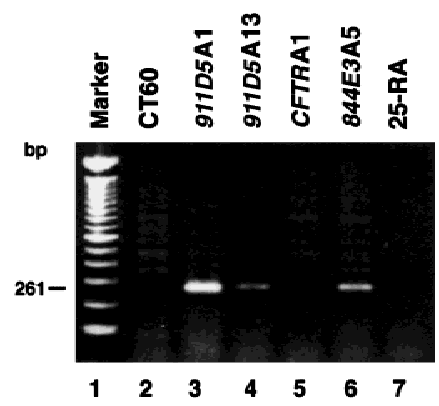

Figure 3 Mapping and expression analysis of the RDA gene fragment 2-R. (A) Ethidium bromide-stained agarose gel of PCR products amplified from the genomic DNA templates listed above lanes 2-9 by use of primers based on the sequence of 2-R. The genomic DNAs are from cell lines unless otherwise indicated. (Lane 1) 100-bp marker; (lane 2) CT60; (lane 3) YAC 844E3; (lane 4) YAC 911D5; (lane 5) 911D5A1; (lane 6) 911D5A13; (lane 7) 911D5B5 [another complemented cell line derived from fusion of CT60 with YAC 911D5 (Gu et al. 1997)]; (lane 8) 844E3A5; (lane 9) $25-\mathrm{RA}$. The expected size of the PCR products is $261 \mathrm{bp}$ as indicated. (B) Ethidium bromide stained agarose gel of RT-PCR products obtained by use of RNA from the cell lines and primers based on the sequence of 2-R. (Lane 1) 100-bp marker; (lane 2) CT60; (lane 3) 911D5A1; (lane 4) 911D5A13; (lane 5) CFTRA1; (lane 6) 844E3A5; (lane 7) 25-RA. The expected size of the RTPCR products is $261 \mathrm{bp}$ as indicated. 


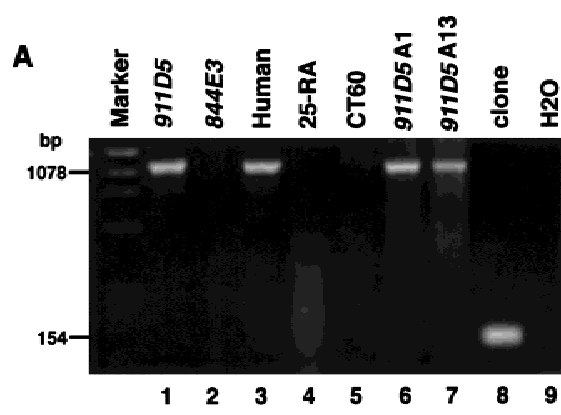

B

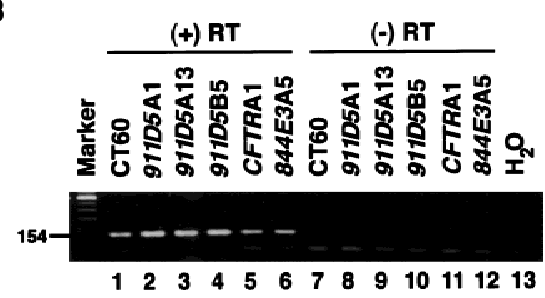

C

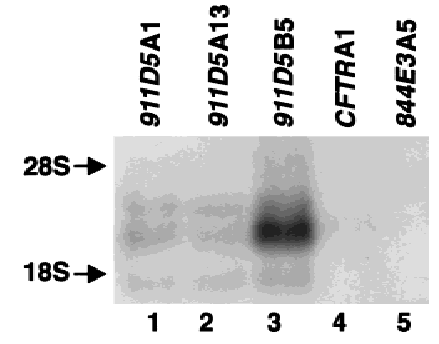

Figure 4 Mapping and expression analysis of the RDA gene fragment 7-R. (A) Ethidium bromide-stained agarose gel of PCR products amplified from the genomic DNA templates by use of primers for 7-R. (Marker) $\phi X$ hae III; (lane 1) YAC 911D5; (lane 2) YAC 844E3; (lane 3) normal human genomic DNA; (lane 4) 25-RA; (lane 5) CT60; (lane 6) 911D5A1; (lane 7) 911D5A13; (lane 8) clone (gene fragment 7-R); (lane 9) water control. The expected size of the PCR product is $154 \mathrm{bp}$ as indicated. (B) Ethidium bromide-stained agarose gel of RT-PCR products obtained using RNA from the cell lines listed and primers for 7-R. (Marker) 100 bp; (lane 1) CT60; (lane 2) 911D5A1; (lane 3) 911D5A13; (lane 4) 911D5B5 [another complemented cell line derived from fusion of CT60 with YAC 911D5 (Gu et al. 1997)]; (lane 5) CFTRA1; (lane 6) 844E3A5; (lanes 7-12) the same samples without addition of RT during CDNA synthesis. The expected size of the RT-PCR products is $154 \mathrm{bp}$ as indicated. (C) Northern blot of RNA from the cell lines listed hybridized with a ${ }^{32} \mathrm{P}$-labeled probe derived from PCR amplification of gene fragment 7-R. (Lane 1) 911D5A1; (lane 2) 911D5A13; (lane 3) 911D5B5; (lane 4) CFTRA1; (lane 5) 844E3A5. Migration of the 28S and 18S RNA are indicated. Subsequent hybridization of this blot with a human GAPDH probe indicated similar loading among all samples except 911D5B5. terestingly, a 261-bp product was also amplified from genomic DNA from YAC $844 E 3$ as well as the cell line 844E3A5 in which $844 E 3$ was stably integrated (Fig. $3 \mathrm{~A})$. YAC $844 E 3$ does not contain NPC1 but overlaps $911 D 5$ as part of the YAC contig encompassing the NP-C locus (Gu et al. 1997). Thus, 2-R maps to the region in which $844 E 3$ and 911D5 overlap. These mapping results were supported by the RT-PCR data that indicated that 2-R was expressed as expected in the testers 911D5A1 and 911D5A13 as well as in 844E3A5, but not in the parental lines CT60 and 25-RA or the driver CFTRA1 (Fig. 3B). Further confirmation of the origin of the 2-R gene fragment was obtained by comparing the sequence of $2-\mathrm{R}$ with the sequences of 911D5 and human genomic DNA. A 223-bp sequence was obtained that was 100\% homologous among 2-R, 911D5, and human genomic DNA (data not shown). Further sequence analysis revealed that gene fragment 2-R did not have significant sequence similarities when BLAST (Altschul et al. 1990) searched against GenBank nonredundant EST division (BLASTN-dbEST), the nonredundant GenBank+EMBL+DDBJ+PDB sequences (BLASTN-NR) and the nonredundant protein sequence databases (BLASTX-NR).

By use of the primers designed to amplify 7-R, PCR products were obtained from genomic DNAs from YAC 911D5, normal human, and 911D5A1 and 911D5A13, but not from the YAC $844 E 3$ or the parental cell lines 25-RA and CT60 (Fig. 4A). Thus, in contrast to 2-R, 7-R did not localize to the overlapping region between $844 E 3$ and 911D5. The PCR products from the genomic DNAs were all the same size but were 900 -bp larger than expected and obtained with gene fragment $7-\mathrm{R}$ as the template ( $154 \mathrm{bp}$; Fig. $4 \mathrm{~A}$, lane 8 ). The data suggested that the 7-R gene fragment spanned more than one exon and that the genomic DNA PCR prod- ucts contained intronic sequence. To assess this issue, RT-PCR and sequence analysis were performed. The RT-PCR data demonstrated the expected 154-bp fragment for each of the hamster cell lines, including the parental line CT60 (Fig. 4B). This data supported the conclusion that gene fragment 7-R spanned more than one exon, but also raised the possibility that expression from the hamster homolog accounted for the detection of 7-R by RDA, even though no product had been obtained by PCR using CT60 genomic DNA. To assess this possibility, the sequence of clone 7-R was compared with the sequences of 911D5 genomic DNA and subcloned RT-PCR products from CT60 obtained using primers designed to amplify 7-R. The data indicated that fragment 7-R was obtained during the RDA procedure as a consequence of expression from a gene on 911D5. There were 84 bp of $100 \%$ homologous sequence between YAC 911D5 and 7-R. Within those 84 $\mathrm{bp}$, there were 11 mismatches in the CT60 sequence compared with 7-R. Further sequence analysis revealed a splice donor site in 911D5 and a splice junction in $7-\mathrm{R}$, supporting the contention that the human 7-R fragment spans more than one exon (data not shown). The lack of amplification of a product from CT60 genomic DNA with 7-R primers (as described above and shown in Fig. 4A) likely reflects that the hamster genomic fragment contains a larger intron than the human DNA.

The sequence of gene fragment 7-R identified a human cDNA clone (Soares ovary tumor NbHOT cDNA clone 741737, GenBank accession no. AA402970, WashU-Merck EST project 1997, unpubl.) with 98\% sequence identity. Therefore, this previously unmapped human cDNA clone was placed on YAC 911D5 on human chromosome 18q11-12 (Gu et al. 1997). Finally, Northern analysis confirmed that 7-R was differ- 
entially expressed in the testers 911D5A1 and 911D5A13 (as well as 911D5B5), compared with the driver, CFTRA1 and the noncomplemented cell line 844E3A5 (Fig. 4C).

As further confirmation of their human chromosomal origin, both 2-R and 7-R were analyzed by radiation hybrid mapping. By use of the Stanford G3 Radiation Hybrid Mapping Panel (webmaster@shgc. stanford.edu) the gene fragments mapped to two neighboring index markers on chromosome $18 \mathrm{q}$ (CHLC.GATA41G05 and SHGC-3938). These markers are separated by $4 \mathrm{cR}$ or $\sim 100 \mathrm{~kb}$, suggesting that the two gene fragments themselves are not far from each other. However, we cannot determine the actual distance between the fragments from this data.

The remaining six RDA gene fragments did not map back to YAC $911 D 5$ (Fig. 2), suggesting that they represented genes upregulated in the hamster genome after YAC transduction. BLAST search showed that three of these fragments had significant sequence similarities with other mammalian genes. The highest nucleotide identity scores were obtained for 5-R (Mus musculus mRNA for Ki-67, 85\% identity), 6-R (rat Y-b3 glutathione $S$-tranferase, $81 \%$ identity), and 8-R (rat cholecystokinin receptor mRNA, 89\% identity).

\section{DISCUSSION}

We have shown that we can directly isolate transcripts expressed from genes present on YACs using complementation cloning and cDNA RDA. Among the isolated transcripts were those from NPC1, which is known to be on YAC 911D5, and two others not localized previously. One of the non-NPC1 fragments, 2-R, is a novel sequence that was further localized during the present study to the region of overlap between two YACs in the NP-C contig used for complementation. The other non-NPC1 fragment, 7-R, spans more than one exon and shares $98 \%$ sequence identity with a previously unmapped human EST. These data suggest the usefulness of this procedure as an adjunct strategy to positional cloning.

This approach has several advantages that complement conventional gene isolation techniques from specific genomic clones: (1) For many regions on human chromosomes, the number of mapped genes or expressed sequence tags (ESTs) is scarce, and thus, database searching and candidate analysis may not be effective. The use of cDNA RDA provides a relatively quick way of identifying exon sequences (Lisitsyn et al. 1993; Hubank and Schatz 1994; Braun et al. 1995; Chu and Paul 1997; Gress et al. 1997), as an addition or alternative to direct selection or techniques such as exon trapping that usually rely on smaller insert clones; (2) RDA theoretically allows genes expressed at relatively low levels to be isolated (Hubank and Schatz
1994). By use of RDA, the absolute abundance of a gene becomes less critical, whereas the degree of difference between the tester and driver cDNAs becomes crucial; (3) large-scale sequencing of genomic DNA for gene identification is still time consuming and costly. cDNA RDA identifies fragments of expressed genes before any sequencing is performed.

The combination of YAC transfer and cDNA RDA has a potential advantage over exon trapping or other techniques in which YACs can be used directly to isolate cDNAs (e.g., direct hybridization to cDNA libraries or cDNA selection). The success of these other methods depends on the presence of specific transcripts in particular cDNA libraries. Without prior knowledge of the tissue-specific expression of the genes on the YAC, there is no easy way to narrow down which libraries to screen. The approach described here does not utilize cDNA libraries or depend on prior knowledge of gene expression patterns: If a gene is on the YAC and is expressed after transfer to mammalian cells, it will be present in the tester representation. On the other hand, if genes contained within a specific YAC are expressed in a cell-type-specific fashion, the cells chosen for transfer of the YAC may be critical to the success of the experiment. This problem could be addressed by utilizing different cell types for YAC transfer and then performing RDA.

Whereas the two goals of the present study were clearly achieved with cDNA RDA, there are potential limitations of this methodology for exhaustive gene isolation. The primary concern is that the RDA gene fragments may represent only a subpopulation of the genes expressed from the YAC. There are several reasons for this limitation. (1) Some of the genuine differences between tester and driver cDNAs may not be selectively amplified if the cDNAs do not contain sufficient sites for the restriction endonuclease used for the initial digestion. (2) The restriction fragments may be too big for efficient amplification by PCR. Therefore, it may be necessary to perform parallel RDA experiments with multiple restriction enzymes to increase the likelihood of obtaining a more complete array of products (Braun et al. 1995). (3) A low yield of novel genes may correlate with the abundance of a single transcript. For example, in the present study, $\sim 75 \%$ of the RDA products obtained were fragments from NPC1. A second RDA in which the driver is supplemented with full-length cDNA of the known abundant transcript might increase the yield of novel genes (Hubank and Schatz 1994).

The flexibility of the RDA methodology that allows for variation in the stringency of hybridization could influence detection of subtle differences in gene expression between tester and driver cDNAs. When difficulties are encountered detecting differential expression, the ratio of the tester and driver cDNAs used to 
generate the difference products becomes critical (Hubank and Schatz 1994). Too much driver cDNA can cause insufficient enrichment of the targets, rendering differences invisible. In contrast, too little driver cDNA may cause insufficient exhaustion of common (but differentially expressed) sequences in the tester cDNA, generating background such as the gene fragments isolated, but not present, in the target YAC 911D5. These fragments could represent genes upregulated in the hamster cells after YAC integration, or genes whose protein products are involved in the same pathway, but downstream to elevated gene expression from the YAC. Given these possibilities, it is crucial to do a detailed assessment of the sequence of the fragments.

Thus, there are several ways to modify cDNA RDA to address problems particular to tester and driver representations generated from YAC transduction of mammalian cells. In the present study, the identification of NPC1 and these two novel non-NPC1 gene fragments suggests that RDA combined with YAC transfer to mammalian cells is useful for gene identification and could be used when only a YAC physical contig exists, but no phenotype is available to monitor. The current strategy is advantageous because it allows for the search for genes to begin at the point that a YAC contig is available, thus accelerating the gene identification process.

\section{METHODS \\ cDNA Synthesis and Generation of Difference Products}

All cell lines were grown as monolayers in Ham's F-12 medium (Biofluids, Rockville, MD) supplemented with 10\% fetal bovine serum (FBS, Hyclone), $2 \mathrm{~mm}$ glutamine, $100 \mathrm{U} / \mathrm{ml}$ penicillin, and $100 \mu \mathrm{g} / \mathrm{ml}$ streptomycin. RNA was extracted from cell monolayers by use of TRIZOL (GIBCO-BRL, Gaithersberg, MD), following the product instructions. Poly(A) ${ }^{+}$ mRNA was separated from total RNA by the Oligo (dT) Columns mRNA Purification Kit (Pharmacia, Piscataway, NJ). Double-stranded cDNA was prepared by reverse transcription by use of the Universal RiboClone cDNA Synthesis System (Promega, Madison, WI).

cDNA representational difference analysis was performed on the basis of the protocol by Hubank and Schatz (1994) with slight modifications. Double-stranded cDNA (2 $\mu \mathrm{g}$ ) was digested with DpnII to generate tester and driver cDNA representations. In the second round of subtraction hybridization, the tester/driver cDNA ratio was increased to 1:40,000 and mung bean nuclease digestion of PCR products was omitted.

\section{Cloning and Characterization of DP II}

Second difference products were directly cloned into pCR 2.1 TA cloning vectors and transformed into $\mathrm{INV}_{\alpha} \mathrm{F}^{\prime}$ One Shot competent cells with the TA Cloning Kit (Invitrogen, Carlsbad, CA). The cloned gene fragments were plated on LB/ Ampicilin plates and colony lifts were prepared for subse- quent screenings with the NPC1 gene or other probes. Representative gene fragments were sequenced and the obtained sequences were BLAST (Altschul et al. 1990) searched against GenBank to identify sequence similarities. PCR primers used to determine the physical locations of the gene fragments were as follows: 2-R-f 5' -CAATCAGAGTGAAGCCTGGGAC-3'; 2-R-r 5'-TCTTGGGGTAGAAACCTACGTCAC-3'; 7-R-f 5'CGTCAAACTCTCCCCGTAACTTG-3'; and 7-R-r 5' CCACGAGAAGGTGCCTGTAAAAAG-3'.

For RT-PCR using 7-R primers, total RNA was treated with RNase-free DNase (RQ1, Promega) at $37^{\circ} \mathrm{C}$ for $30 \mathrm{~min}$, and then at $90^{\circ} \mathrm{C}$ for $10 \mathrm{~min}$ to inactivate the DNase. Reverse transcription and PCR were performed by the SUPERSCRIPT Preamplification System (GIBCO-BRL, Gaithersburg, MD) following the manufacturer's instructions.

\section{DNA Sequencing}

DNA fragments were amplified by PCR from human genomic DNA and YAC 911D5 with the primer pairs, 2-R-f/2-R-r and 7-R-f/7-R-r, gel purified and directly sequenced by automated sequencing. DNA fragments amplified by RT-PCR from CT60 by use of the same primers were cloned into T-Adv vector (Clontech, Palo Alto, CA). Six CT60 clones containing the 2-R insert and eight clones containing the $7-\mathrm{R}$ insert were sequenced by automated sequencing. Sequences were aligned by the MacVector 6.0.1 Align program (Oxford Molecular, Ltd.).

\section{Northern Blot Analysis}

For each RNA sample, $10 \mu$ g was loaded on a $1.2 \%$ agarose/ formaldehyde gel. Northern blotting and hybridization were performed as described (Mogayzel et al. 1996). Probes were labeled by random oligo extension (Ready-To-Go kit, Pharmacia Biotech), following the kit instructions. After a 10-min denaturation period, repeated sequences were blocked by incubating the probe with unlabeled human placental and Cot- 1 DNA at $65^{\circ} \mathrm{C}$ for $30 \mathrm{~min}$.

\section{ACKNOWLEDGMENTS}

We thank L. Staudt (National Cancer Institute) for providing a protocol for cDNA RDA, F. Collins, J. Trent, D. Tagle, and S. Chandrasekharappa for their contributions to this study, K. Henning, P. Mogayzel, and P. Liu for critical reading of the manuscript, B. Pike for performing the radiation hybrid mapping, P. Pentchev, E. Carstea, and J. Morris (National Institute of Neurological Disorders and Stroke) for providing the NPC1 full-length cDNA probe, J. Morris and E. Carstea for providing unpublished data, and D. Leja for graphics assistance. J.G. was supported by a grant from the Ara Parseghian Medical Research Foundation.

The publication costs of this article were defrayed in part by payment of page charges. This article must therefore be hereby marked "advertisement" in accordance with 18 USC section 1734 solely to indicate this fact.

\section{REFERENCES}

Altschul, S.F., W. Gish, W. Miller, E.W. Myers, and D.J. Lipman. 1990. Basic local alignment search tool. J. Mol. Biol. 215: 403-410. 
Gu et al.

Braun, B.S., R. Frieden, S.L. Lessnick, W.A. May, and C.T. Denny. 1995. Identification of target genes for the Ewing's Sarcoma EWS/FLI fusion protein by representational difference analysis. Mol. Cell. Biol. 15: 4623-4630.

Buckler, A.J., D.D. Chang, S.L. Graw, J.D. Brook, D.A. Haber, P.A Sharp, and D.E. Housman. 1991. Exon amplification: A strategy to isolate mammalian genes based on RNA splicing. Proc. Natl. Acad. Sci. 88: 4005-4009.

Cadigan, K.M., D.M. Spillane, and T-Y. Chang. 1990. Isolation and characterization of Chinese hamster ovary cell mutants defective in intracellular low density lipoprotein-cholesterol trafficking. $L$. Cell Biol. 110: 295-308.

Carstea, E.D., J.A. Morris, K.G. Coleman, D. Zhang, C. Cummings, J. Gu, M.A. Rosenfeld, S.K. Loftus, W.J. Pavan, D.B. Krizman et al. 1997. Cloning the Niemann-Pick C gene: Homology to mediators of cholesterol homeostasis. Science 27 7: 228-231.

Chu, C.C. and W.E. Paul. 1997. Fig1, an interleukin 4-induced mouse B cell gene isolated by cDNA representational difference analysis. Proc. Natl. Acad. Sci. 94: 2507-2512.

Del Mastro, R.G. and M. Lovett. 1997. Isolation of coding sequences from genomic regions using direct selection. Methods Mol. Biol. 68: $183-199$.

Duyk, G.M., S.W. Kim, R.M. Myers, and D.R. Cox. 1990. Exon Trapping: A genetic screen to identify candidate transcribed sequences in cloned mammalian genomic DNA. Proc. Natl. Acad. Sci. 87: 8995-8999.

Gress, T.M., C. Wallrapp, M. Frohme, F. Muller-Pillasch, U. Lacher, H. Friess, M. Buchler, G. Adler, and J.D. Hoheisel. 1997. Identification of genes with specific expression in pancreatic cancer by cDNA representational difference analysis. Genes, Chromosomes, Cancer 19: 97-103.

Gu, J.Z., E.D. Carstea, C. Cummings, J.A. Morris, S.K. Loftus, K.G.
Coleman, D. Zhang, A. Cooney, M. Comly, L. Fandino et al. 1997. Substantial narrowing of the Niemann-Pick C candidate interval by yeast artificial chromosome complementation. Proc. Natl. Acad. Sci. 94: 7378-7383.

Hubank, M. and D.G. Schatz. 1994. Identifying differences in mRNA expression by representational difference analysis of cDNA. Nucleic Acid Res. 22: 5640-5648.

Krizman, D.B. and S.M. Berget. 1993. Efficient selection of 3'-terminal exons from vertebrate DNA. Nucleic Acids Res. 21: 5198-5202.

Lisitsyn, N., N. Lisitsyn, and M. Wigler. 1993. Cloning the differences between two complex genomes. Science 259: 946-951.

Lovett, M. 1994. Fishing for complements: Finding genes by direct selection. Trends Genet. 10: 352-357.

Mogayzel, P.J., K.A. Henning, M.L. Bittner, E.A. Novotny, E.M. Schwiebert, W.B. Guggino, Y. Jiang, and M.A. Rosenfeld. 1997 Functional human CFTR produced by stable Chinese hamster ovary cell lines derived using yeast artificial chromosomes. Hum. Mol. Genet. 6: 59-68.

Osborne-Lawrence, S., P.L. Welsh, M. Spillman, S.C. Chandrasekharappa, T.D. Gallardo, M. Lovett, and A.M. Bowcock. 1995. Direct selection of expressed sequences within a $1-\mathrm{Mb}$ region flanking BRCA1 on human chromosome 17q21. Genomics 25: 248-255.

Simmons, A.D., S.A. Goodart, T.D. Gallardo, J. Overhauser, and M. Lovett. 1995. Five novel genes from the cri-du-chat critical region isolated by direct selection. Hum. Mol. Genet. 4: 295-302.

Received September 11, 1998; accepted in revised form December 5, 1998.

188 Genome Research 


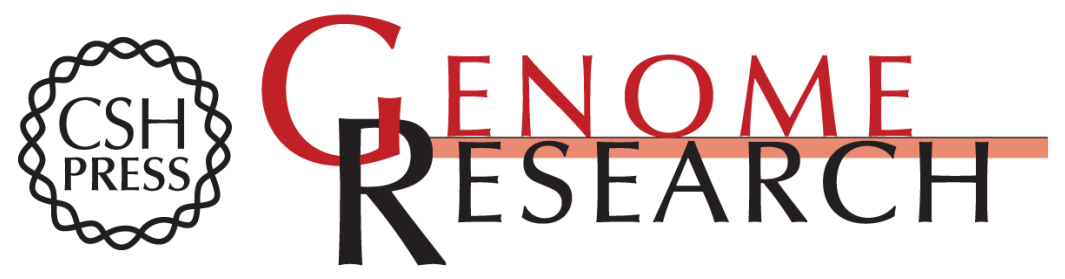

\section{Isolation of Human Transcripts Expressed in Hamster Cells from YACs by cDNA Representational Difference Analysis}

Jessie Gu, Xin-Yuan Guan and Melissa A. Ashlock

Genome Res. 1999 9: 182-188

Access the most recent version at doi:10.1101/gr.9.2.182

References This article cites 17 articles, 8 of which can be accessed free at:

http://genome.cshlp.org/content/9/2/182.full.html\#ref-list-1

\section{License}

Email Alerting Receive free email alerts when new articles cite this article - sign up in the box at the Service top right corner of the article or click here.

\section{Affordable, Accurate Sequencing.}

